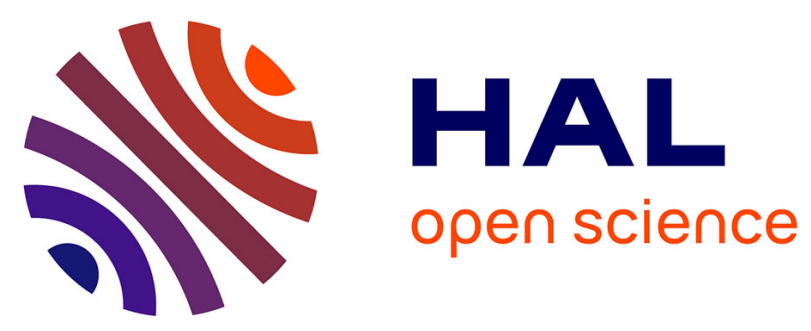

\title{
Hemisyntheses and In-Silico Study of New Analogues of Carlina Oxide from Carthamus Caeruleus Roots
}

\author{
Imane Rihab Mami, Tabet Zatla Amina, Joëlle Pérard, Zoheir Arrar, \\ Mohammed El Amine Dib
}

\section{- To cite this version:}

Imane Rihab Mami, Tabet Zatla Amina, Joëlle Pérard, Zoheir Arrar, Mohammed El Amine Dib. Hemisyntheses and In-Silico Study of New Analogues of Carlina Oxide from Carthamus Caeruleus Roots. Combinatorial Chemistry and High Throughput Screening, 2021, 24, pp.1503-1513. 10.2174/1386207323999201103214141 . hal-03186433

\section{HAL Id: hal-03186433 https://hal.science/hal-03186433}

Submitted on 8 Apr 2021

HAL is a multi-disciplinary open access archive for the deposit and dissemination of scientific research documents, whether they are published or not. The documents may come from teaching and research institutions in France or abroad, or from public or private research centers.
L'archive ouverte pluridisciplinaire HAL, est destinée au dépôt et à la diffusion de documents scientifiques de niveau recherche, publiés ou non, émanant des établissements d'enseignement et de recherche français ou étrangers, des laboratoires publics ou privés. 


\section{Hemisyntheses and In-Silico Study of New Analogues of Carlina Oxide from Carthamus Caeruleus}

\section{Roots}

\section{Imane Rihab Mami ${ }^{1}$, Tabet Zatla Amina ${ }^{1}$, Joëlle Pérard ${ }^{2}$, Zoheir Arrar ${ }^{1}$, Mohammed El Amine Dib $^{3 *}$}

${ }^{1}$ Laboratoire de chimie organique, substances naturelles et analyses (COSNA), Université de Tlemcen, Algérie

${ }^{2}$ Université Paris Descartes, Faculté de Pharmacie, CNRS (UMR 8038)

${ }^{3}$ Laboratoire des Substances Naturelles et Bioactives (LASNABIO), Université de Tlemcen, BP 119, 13000, Algérie, E-mail a_dibdz@yahoo

Abstract: Aim and Objective: Nowadays, developing effective antibiotics for bacteria control has become difficult due to increased resistance to the available medicines in the market. Essential oils have very interesting biological properties; some of their components have very powerful antiviral and antibacterial properties. Carthamus caeruleus is a plant that has antibacterial and antioxidant activity due to the presence of an acetylenic compound, Carlina oxide. The aim of this work was to provide for the first time the chemical modifications to the structure of Carlina oxide and the in-silico study of these analogues.

Materials and Methods: The essential oil of Carthamus caeruleus was extracted by steam distillation in a Clevenger-type apparatus. Carlina oxide component was separated by column chromatography. Five new analogues were synthetized and identified by spectroscopic analyses (RMN, IR and SM). Molecular docking simulation study was performed using Molecular Operating Environment software (MOE) on three enzymes of bacterial origin (Streptococcus pyogenesis and Enterococcus faecalis).

Results: Five new compounds derived from Carlina oxide were synthesized (IM8-IM12), and their structures were characterized by infrared (IR), $1 \mathrm{H}$ and 13C nuclear magnetic resonance (NMR). The new synthesized compounds were evaluated as mSpeB, DHFR from Enterococcus faecalis and DNA gyrase inhibitors by a docking analysis using MOE. These results show interesting ligand interactions with the three enzymes, and the best result was attributed to the complexes formed with IM9, which had the lowest score.

Conclusion: In fact, these new compounds could lead to powerful approaches for the research anddevelopment of new antibiotics.

Keywords: Carlina oxide, Vilsmeier -Haack reaction, reductive amination. Molecular docking. Enterococcus faecalis, DNA gyrase inhibitors.

\section{Introduction}

Over time, the massive and repeated use of antibiotics creates various forms of increased bacterial resistance that threaten the effectiveness of treatments. So, many infections are becoming increasingly 
difficult to treat, such as pneumonia, gonorrhea, tuberculosis and even salmonellosis and are one of the major causes of the increased mortality of the global population [1]. The success of plant-based products such as paclitaxol in the treatment of tumors or artemisinin in the treatment of malaria has given the impetus for the introduction of numerous research projects, particularly in Pharmaceutical industry [2-4]. Considerable efforts are being made to generate new molecules of natural origin (plant, marine, microbial, etc.) $[5]$.

Formerly, polyacetylenes were not the kind of compounds that are expected to be found in nature. Nowadays, in addition to the overwhelming evidence that they are produced, over the past few years there has been a growing body of evidence on how they are produced [6]. Acetylene includes all substances with a triple carbon-carbon bond or alkynyl functional groups, which are chemically and biologically active. Due to their high reactivity they can be degraded or oxidized under certain conditions such as UV light or $\mathrm{pH}$ of the medium, which characterizes them as unstable product, necessitating particular extraction and investigation conditions 7.

These compounds may contain heteroatoms, such as oxygen, sulphur and nitrogen, it includes aliphatic structures and various cyclic structures [8,9]. Acetylenes are widespread in nature found in living materials and form distinguishable group of chemically and biologically active natural compounds, which have been detected in numerous higher plant families. More than 1100 different acetylene have been extracted and identified in species of the Asteraceae family [9-13].

Carlina oxide is an acetylenic compound which was isolated for the first time from Carlina accaulis by Semmler in 1902 [14] and its correct structure was proposed by Gilman van ess and Burtner in 1933 [15]. Subsequently, it has been confirmed that Carlina oxide is present in the chemical composition of several extracts and essential oils of plants belonging to the Asteraceae family, it is present mainly in the essential oil of Carlina accaulis [14-16], Carlina acanthifolia [17], Carlina diae [18], Carlina vulgaris [19], Atractylis gummifera [20] and Carthamus caeruleus [21], which gives them potential biological effects.

Several studies have been conducted on the different biological activities provided by this compound which has shown very promising antimicrobial, antitrypanosmal, anti-inflammatory, antiulcer and antioxidant activities [19-24]. On the other hand, the study by Benelli et al [25] showed that carlina oxide exhibits a certain non-selective cytotoxicity towards normal human dermis cells and tumor cell lines. The toxicity of Carlina oxide is partly due to the inhibition of the enzyme acetylcholinesterase [26,27]. Carthamus caeruleus is a plant that is traditionally used as a healing agent, they help to heal burns and treat skin diseases [27], it is also used against joint inflammations and is very effective against diseases like syndrome colon irritable for cancer patients. In the previous study, we have shown that the essential oil of the roots of Carthamus caeruleus was rich of Carlina oxide with a percentage exceeding 80\% [21]. 
To our knowledge, no work has reported the synthesis of analogues of carlina oxide and the study of their biological activities. The main objective of this work consisted to prepared by hemisynthesis five new analogues of Carlina oxide and study their in-silico antimicrobial activity against Streptococcus pyogenesis and Enterococcus faecalis.

\section{MATERIALS AND METHODS}

\subsection{General Information}

All chemical reagents and solvent used in this study were purchased from commercial sources (Aldrich and Fluka) as reagents of certified quality and used without any further purification. The infrared spectra were monitored on Agilent technologies cary 600 series FTIR. ${ }^{1} \mathrm{H}$ NMR experiments were performed in deuterated chloroform $\left(\mathrm{CDCl}_{3}\right)$. Compounds spectra were recorded on a Bruker Avance $300 \mathrm{MHz}$ using the TMS as an internal standard. The chemical shifts were referenced to the residual solvent signal and expressed in ppm. All compounds have been regularly checked by TLC and ${ }^{1} \mathrm{H}$ NMR. The TLC were performed on Fluka $\mathrm{F}_{254}$ silica gel and visualized under light at $254 \mathrm{~nm}$ or by revelation of phosphomolybdic acid. Silica gel (60A, 70-230 mesh, 63-200 $\mu \mathrm{m}$ Fluka) was used for column chromatography. After the extractions and reactions, the solvent was removed using a rotary evaporator under reduced pressure.

\subsection{Plant material, extraction of essential oil and Isolation of carlina oxide}

Roots of Carthamus caeruleus were harvested from Beni snous station near Tlemcen city (Algeria) in May 2017. The essential oil of air-dried roots of Carthamus caeruleus were extracted by hydrodistillation in a Clevenger-type apparatus, according to the European Pharmacopoeia. Carlina oxide was isolated using silica gel column chromatography (FC, silica gel 200-500 mm) eluted by hexane/EtOAc (3:1).

\subsection{General procedure for the formylation of Carlina oxide (IM2)}
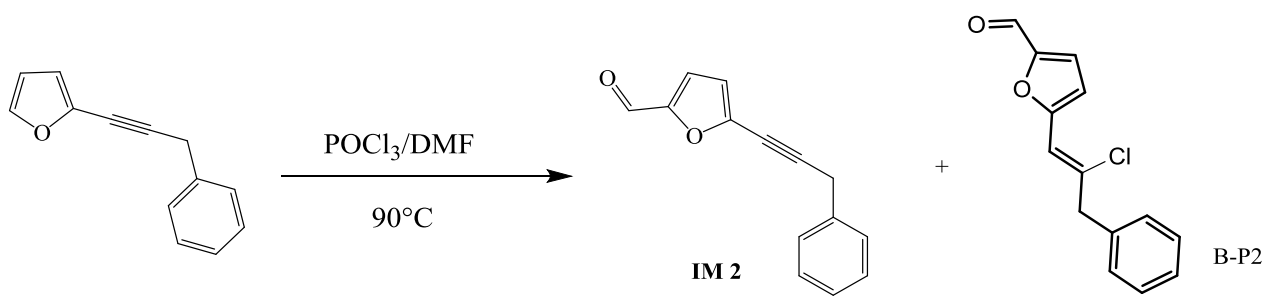

Scheme 1 : Vilsmeier haak formylation of CarOx

Method 1: $0.85 \mathrm{ml}$ of freshly distilled $\mathrm{POCI}_{3}$ was added dropwise to $0.8 \mathrm{ml}$ of anhydrous DMF at $0{ }^{\circ} \mathrm{C}$, the mixture was left for 20 min under external cooling. Carlina oxide $(8.23 \mathrm{mmol})$ were added slowly. The mixture was stirred for 45 minutes at $90{ }^{\circ} \mathrm{C}$ and cooled on ice. The resulting mixture was neutralized with $60 \% \mathrm{~K}_{2} \mathrm{CO}_{3}$. The organic phase was extracted 3 times with chloroform and dried under $\mathrm{MgSO}_{4}$. After 
vacuum elimination of the solvent, the residue obtained in a black paste-like was purified by chromatographic column on silica gel with a mixture of cyclohexane-diethyl ether (80:20).

Method 2: In order to remove the $\mathrm{HCl}$ produced during the formylation reaction, a gas trap was employed and a few drops of triethylamine were added to the reaction mixtures under the same conditions as in method 1.

\subsection{General procedure for the preparation of IM8, IM10, IM11}

IM2 (0.5 mmol) and corresponding amino acid ester hydrochloride (5eq) were dissolved in $\mathrm{CH}_{3} \mathrm{OH}(15$ $\mathrm{mL}$ ), then $\mathrm{NaBH}_{3} \mathrm{CN}$ (2eq) was added. The mixture was then stirred at room temperature until the starting material disappeared. The evolution of the reaction was monitored by thin layer chromatogrphy (TLC). At the end of the reaction the solvent was removed, the residue was washed with distilled water and extracted 3 times with diethyl ether. The combined organic phases were then dried on $\mathrm{MgSO}_{4}$ and filtered. After removal of the solvent, the residue was purified by column chromatography using diethyl ethercyclohexane (60:40), (45:55), (45:55) to give the corresponding product (Scheme 2).
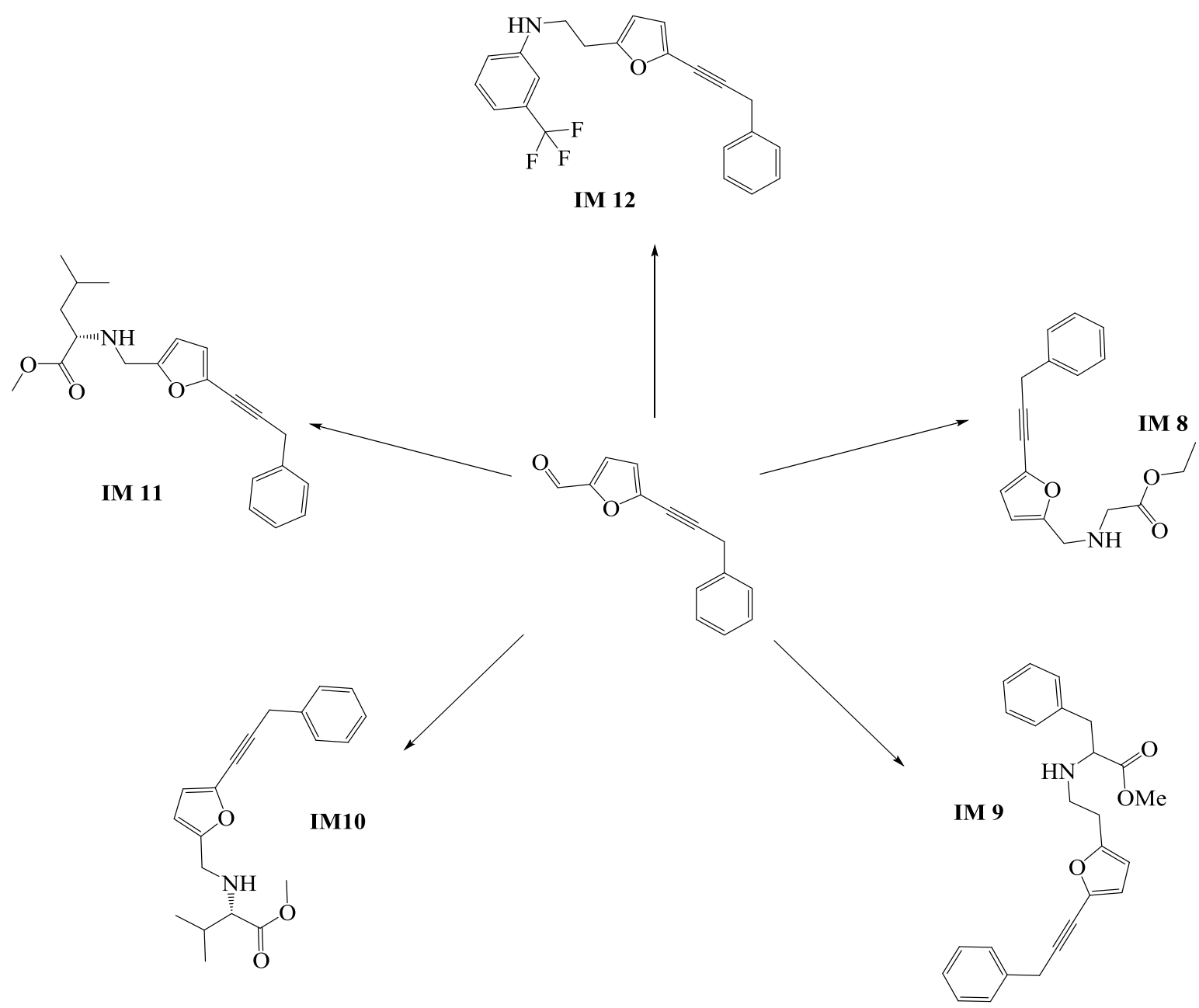

Scheme 2: CarOx analogues hemisynthesis 


\subsection{General procedure for the preparation of IM9}

L-Phenylalanine methyl ester hydrochloride (1eq) and IM2 (0.5 mmol) were dissolved in $\mathrm{CH}_{3} \mathrm{OH}(15 \mathrm{~mL})$ once all is dissolved $\mathrm{NaBH}_{3} \mathrm{CN}$ (2eq) was added. The mixture was then stirred at room temperature. The reaction was followed by TLC. At this stage, the reaction has been treated in the same manner as the IM8 product. The residue was purified by column chromatography with cyclohexane-ether diethyl (55:45) (Scheme 2).

\subsection{General procedure for the preparation of IM 12}

IM2 (1 mmol) and 3-(Trifluoromethyl) aniline (1eq) were dissolved in $\mathrm{CH}_{3} \mathrm{OH}$ to which a few drops of acetic acid were added. $\mathrm{NaBH}_{3} \mathrm{CN}$ (1eq) was subsequently added under agitation. The reaction took place at room temperature and was followed by TLC. At the end of the reaction, the solvent has been evaporated and the residue has undergone the same treatment as the previous compounds. The raw product was purified by column chromatography using a mixture of cyclohexane-ether diethyl (80:20) as eluent (Scheme 2).

\subsection{Preparation and Optimization of Both Enzymes and Inhibitors}

Molecular docking simulation study was performed using Molecular Operating Environment software (MOE) [28]. The energy minimization was carried out under the following conditions: Temperature $=300$ ${ }^{\circ} \mathrm{K}, \mathrm{pH}=7$, geometry was realized using the field values of MMFF94x and Hamiltonian AM1. The X-ray crystallographic structure of the mature streptococcal cysteine protease, mSpeB, DHFR from Enterococcus faecalis, and Escherichia coli DNA gyrase (PDB: 2UZJ, 4M7U, 1KZN) respectively, was downloaded from the protein data bank. The water molecules were removed and the receptor was prepared for the docking study. A triangular matcher algorithm with rescoring GBVI/WSA dG were applied. Synthetized compound structures were built and registered in their 3D conformation.

Minimized energy of ligands and their toxicity are shown in table 1. Figure 1 shows the active site of the enzymes with molecule of co-crystallization.

Table 1: ligands properties and energy minimization of carlina oxide and synthesized analogues

\begin{tabular}{cccccccc}
\hline Ligands & $\begin{array}{c}\text { Molecular weight } \\
\text { g/mol }\end{array}$ & Toxicity & \multicolumn{2}{c}{ TPSA } & Log P & Log S & Energie (Kcal/mol) \\
& & & H donn & H acc & & & \\
\hline CarOx & 182.22 & no & 0 & 0 & 2.87 & -4.18 & -2888.18710 \\
\hline IM8 & 311.38 & no & 1 & 2 & 3.18 & -5.01 & -3902.2628 \\
\hline IM9 & 373.45 & no & 1 & 2 & 4.01 & -6.31 & -5825.1593 \\
\hline IM10 & 325.41 & no & 1 & 2 & 3.43 & -5.21 & -4895.31 \\
\hline IM11 & 339.43 & no & 1 & 2 & 3.82 & -6.04 & -5175.16 \\
\hline IM12 & 355.36 & no & 1 & 0 & 6.08 & -6.95 & -4667.8431 \\
\hline
\end{tabular}



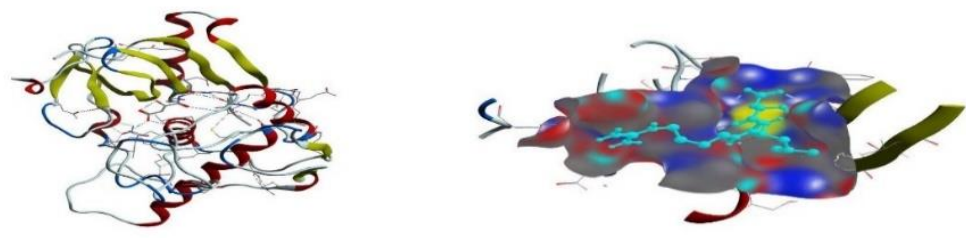

a:

Mature Streptococcal cysteineprotease mSpeB (2UZJ)
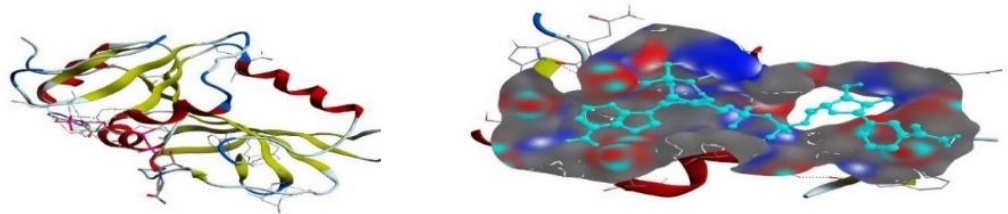

b: Dihydrofolatereductase DHFR from Enterococcus faecalis (4M7U)
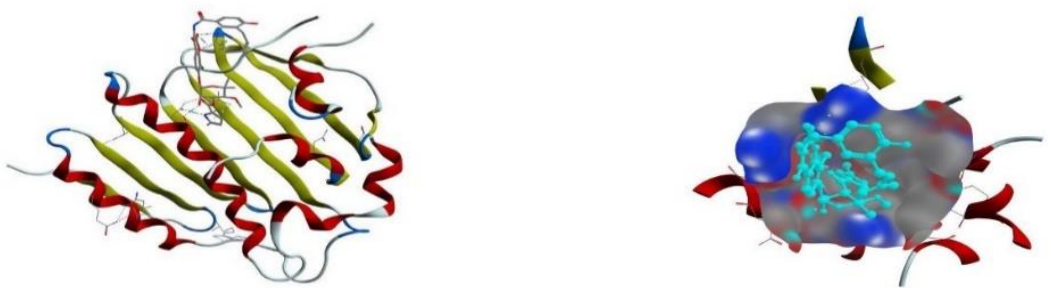

c : DNA gyrase (1KZN)

Fig 1: Simplified model of enzymes and site active isolated

\subsection{Docking and Building Complexes}

The validated docking protocol was used to study ligand receptor interactions at the active site of the newly synthesized compounds to predict their binding mode and affinity. Following the formation of the ligand-receptor complex, it will adapt the most stable conformation according to the lowest energy level. The results were finally examined to determine the most potent and effective antibacterial inhibitor by visualizing various ligand interactions in the binding pocket.

\section{Results and discussion}

\subsection{Chemistry}

The steps elaborated during this work were, in beginning, the formylation of Vilsmeier-Haack on the furano group of Carlina oxide, in order to have an aldehyde (Scheme 1). Thereafter the aldehyde was used to create an $\mathrm{N}-\mathrm{C}$ bond by a reductive amination by using amino acid ester hydrochloride to obtain the corresponding analogues (Scheme 2). The desired compounds (IM2, IM8, IM9, IM10, IM11, IM12) were obtained mixed with by-products (B-P2, B-P8, B-P9, B- 
P10, B-P11, B-P12) respectively. The by-products could not be eliminated due to the same Rf of the desired product (Scheme 3).

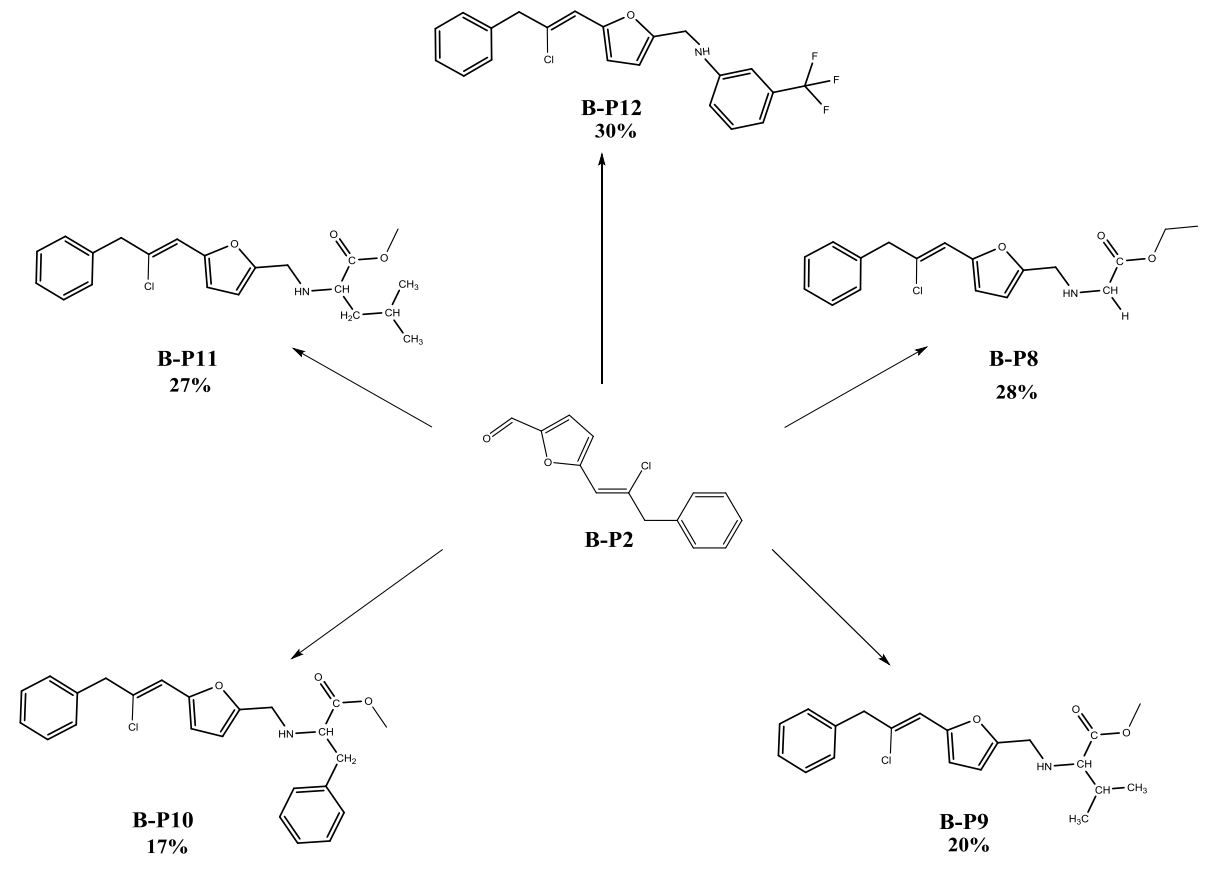

Scheme 3: by-product of reductive amination

\section{5-(3-Phenyl-prop-1-ynyl)-furan-2-carbaldehyde (IM2)}

As shown in the scheme 1, the formylation reaction of vilsmeir haack gave a mixture of two inseparable compounds per chromatographic column whose appearance is a yellow oil with a yield of $64 \%$ by method 1 and $67 \%$ by method 2 .

IM2: (method1): 70\%. (method2): 90\%. IR spectrum (KBr, $\left.\mathrm{cm}^{-1}\right) 1678(\mathrm{C}=\mathrm{O}$, Aldehyde), 2825(C-H, aldehyde), 2229 (C $\equiv \mathrm{C}), 1590,1485 ;{ }^{1} \mathrm{H} \mathrm{RMN}\left(\mathrm{CDCl}_{3}, 300 \mathrm{MHz}\right), \delta, \mathrm{ppm}(\mathrm{J}, \mathrm{Hz})$ : $9.64(\mathrm{~s}, 1 \mathrm{H}), 7.42-7.30(\mathrm{~m}, 5 \mathrm{H}) 7.25(\mathrm{~d}, 1 \mathrm{H} \mathrm{J}=3.6 \mathrm{~Hz}), 6.70(\mathrm{~d}, 1 \mathrm{H}, \mathrm{J}=3.6 \mathrm{~Hz}), 3.92(\mathrm{~s}, 2 \mathrm{H}, \mathrm{CH} 2)$, ${ }^{13} \mathrm{C}$ NMR (300 MHz, $\mathrm{CDCl}_{3}$ ) $\delta: 177.19,152.14,142.19,134.99,128.81,128.04,127.14,116.44$, 112.35, 95.94, 72.22, 25.90 .

B-P2: (method1): 30\%; (method2): 10\% IR spectrum $\left(\mathrm{KBr}, \mathrm{cm}^{-1}\right) 697(\mathrm{C}-\mathrm{Cl}) .{ }^{1} \mathrm{H}$ RMN $\left(\mathrm{CDCl}_{3}\right.$, $300 \mathrm{MHz}), \delta$, ppm (J, Hz): 9.64 (s, 1H),7.42-7.30(m, 5H ar + H furano), 7.16 (d, 1H, J = 3.9Hz),

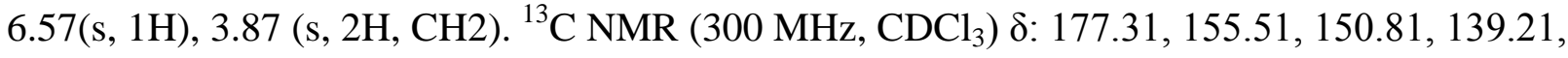
$136.07,129.27,128.86,127.18,123.24,115.51,112.61,47.04$.

\{[5-(3-Phenyl-prop-1-ynyl)-furan-2-ylmethyl]-amino\}-acetic acid ethyl ester (IM8) 
Glycine ethyl ester hydrochloride has produced $59.4 \mathrm{mg}$ (40\%) of orange oil composed of IM8 and by product B-P8.

IM8: 72\% IR spectrum $\left(\mathrm{KBr}, \mathrm{cm}^{-1}\right) 3340(\mathrm{~N}-\mathrm{H}), 2230(\mathrm{C} \equiv \mathrm{C}) .1737(\mathrm{C}=\mathrm{O}$ ester $),{ }^{1} \mathrm{H}$ NMR $(300$ $\left.\mathrm{MHz} \mathrm{CDCl}_{3}\right) \delta 7.27-7.44(\mathrm{~m}, 5 \mathrm{H}), 6.50(\mathrm{~d}, J=3.3 \mathrm{~Hz}, 1 \mathrm{H}), 6.23(\mathrm{~d}, J=3.3 \mathrm{~Hz}, 1 \mathrm{H}), 4.23(\mathrm{q}, J$ $=7.1 \mathrm{~Hz}, 2 \mathrm{H}), 3.89(\mathrm{~s}, 2 \mathrm{H}), 3.85(\mathrm{~s}, 2 \mathrm{H}), 3.46(\mathrm{~s}, 2 \mathrm{H}), 2.08(\mathrm{~s}, 1 \mathrm{H}), 1.32(\mathrm{t}, J=7.1 \mathrm{~Hz}, 3 \mathrm{H}) .{ }^{13} \mathrm{C}$ NMR (300 MHz, $\left.\mathrm{CDCl}_{3}\right) \delta: 172.05,153.80,136.92,136.80,128.65,128.03,126.83,115.05$, $108.57,92.05,73.05,60.90,49.78,45.66,25.86,14.24$.

B-P8: 28\%. IR spectrum $\left(\mathrm{KBr}, \mathrm{cm}^{-1}\right) 698(\mathrm{C}-\mathrm{Cl}){ }^{1} \mathrm{H}$ NMR $\left(300 \mathrm{MHz}, \mathrm{CDCl}_{3}\right) \delta 7.27-7.44(\mathrm{~m}$, $5 \mathrm{H}), 6.89(\mathrm{~d}, J=3.4 \mathrm{~Hz}, 1 \mathrm{H}), 6.48(\mathrm{~s}, 1 \mathrm{H}), 6.30(\mathrm{~d}, J=3.4 \mathrm{~Hz}, 1 \mathrm{H}), 4.23(\mathrm{q}, J=7.1 \mathrm{~Hz}, 2 \mathrm{H}), 3.85$ $(\mathrm{s}, 2 \mathrm{H}), 3.81(\mathrm{~s}, 2 \mathrm{H}), 3.46(\mathrm{~s}, 2 \mathrm{H}), 2.08(\mathrm{~s}, 1 \mathrm{H}), 1.32(\mathrm{t}, J=7.5 \mathrm{~Hz}, 3 \mathrm{H}) .{ }^{13} \mathrm{C} \mathrm{NMR}(300 \mathrm{MHz}$, $\left.\mathrm{CDCl}_{3}\right) \delta: 172.11,151.97,149.97,136.74,131.80,129.18,128.65,127.10,116.11,111.05$, $109.7,60.90,49.72,46.64,25.86,14.24$.

\section{3-Phenyl-2-\{[5-(3-phenyl-prop-1-ynyl)-furan-2-yl meth-yl]-amino\}-propionic acid methyl ester (IM9)}

Using phenylalanine methyl ester hydrochloride, a mixture of IM9 and B-P9 was obtained. Appearance: orange oil. Yield: 30\%.

IM9: 80\%. IR spectrum $\left(\mathrm{KBr}, \mathrm{cm}^{-1}\right) 3338(\mathrm{~N}-\mathrm{H}), 2232(\mathrm{C} \equiv \mathrm{C}) .1734(\mathrm{C}=\mathrm{O}$ ester $){ }^{1} \mathrm{H} \mathrm{RMN}$ $\left(\mathrm{CDCl}_{3}, 300 \mathrm{MHz}\right), \delta$, ppm (J, Hz): ${ }^{1} \mathrm{H}$ NMR (300 MHz, $\left.\mathrm{CDCl}_{3}\right) \delta 7.43-7.06(\mathrm{~m}, 10 \mathrm{H}), 6.47$ (d, $J=3.3 \mathrm{~Hz}, 1 \mathrm{H}), 6.11(\mathrm{~d}, J=3.3 \mathrm{~Hz}, 1 \mathrm{H}), 3.90(\mathrm{~s}, 2 \mathrm{H}), 3.80(\mathrm{~s}, 3 \mathrm{H}), 3.69(\mathrm{~s}, 2 \mathrm{H}), 3.62(\mathrm{t}, J=6.9$ $\mathrm{Hz}, 1 \mathrm{H}), 3.02(\mathrm{~d}, J=20 \mathrm{~Hz}, 2 \mathrm{H}) 2.01(\mathrm{~s}, 1 \mathrm{H}) .{ }^{13} \mathrm{C} \mathrm{NMR}\left(300 \mathrm{MHz}, \mathrm{CDCl}_{3}\right) \delta: 174.54,153.82$, 137.02, 136.65, 129.18, 128.67, 128.51, 128.05, 126.85, 115.06, 108.42, 91.98, 73.10, 61.79, $51.84,44.78,39.61,25.89$.

B-P9: $20 \%$.IR spectrum $\left(\mathrm{KBr}, \mathrm{cm}^{-1}\right) 699(\mathrm{C}-\mathrm{Cl}) .{ }^{1} \mathrm{H} \mathrm{RMN}\left(\mathrm{CDCl}_{3}, 300 \mathrm{MHz}\right), \delta, \mathrm{ppm}(\mathrm{J}, \mathrm{Hz}):{ }^{1} \mathrm{H}$ NMR $\left(300 \mathrm{MHz}, \mathrm{CDCl}_{3}\right) \delta 7.43-7.06(\mathrm{~m}, 10 \mathrm{H}), 6.86(\mathrm{~d}, J=3.3 \mathrm{~Hz}, 1 \mathrm{H}), 6.44(\mathrm{~s}, 1 \mathrm{H}), 6.19(\mathrm{~d}, J$ $=3.3 \mathrm{~Hz}, 1 \mathrm{H}), 3.85(\mathrm{~s}, 2 \mathrm{H}), 3.82(\mathrm{~s}, 3 \mathrm{H}), 3.68(\mathrm{~s}, 2 \mathrm{H}), 3.62(\mathrm{t}, J=6.9 \mathrm{~Hz}, 1 \mathrm{H}), 3.02(\mathrm{~d}, J=20$ $\mathrm{Hz}, 2 \mathrm{H}), 2.01$ (s, $1 \mathrm{H}) .{ }^{13} \mathrm{C} \mathrm{NMR}\left(300 \mathrm{MHz} \mathrm{CDCl}_{3}\right) \delta: 174.51,153.82,137.02,136.65,135.98$, $129.18,128.52$, 127.18, 126.85, 115.06, 111.06, 108.6, 61.79, 51.84, 44.79, 39.61, 25.89.

\section{3-Methyl-2-\{[5-(3-phenyl-prop-1-ynyl)-furan-2-ylmethyl]-amino\}-butyric acid methyl ester (IM10)}


- A yellow oil composed of IM10 and B-P10 was obtained using Valine methyl ester hydrochloride with a yield $35 \%$

IM 10: 83\%.IR spectrum $\left(\mathrm{KBr}, \mathrm{cm}^{-1}\right) 3338(\mathrm{~N}-\mathrm{H}), 2232(\mathrm{C} \equiv \mathrm{C}) .1734(\mathrm{C}=\mathrm{O} \text { ester })^{1} \mathrm{H} \mathrm{RMN}$ $\left(\mathrm{CDCl}_{3}, 300 \mathrm{MHz}\right), \delta, \operatorname{ppm}(\mathrm{J}, \mathrm{Hz}): \delta 7.42-7.30(\mathrm{~m}, 5 \mathrm{H}), 6.50(\mathrm{~d}, J=3.4 \mathrm{~Hz}, 1 \mathrm{H}), 6.20(\mathrm{~d}, J=$ $3.2 \mathrm{~Hz}, 1 \mathrm{H}), 3.90$ (s, 2H), 3.81 (s, 2H), 3.74 (s, 3H), 3.11 (d, J= 5.7 Hz, 1H), 1.98 (h, J= 13.4, $\left.6.7 \mathrm{~Hz}, 1 \mathrm{H}^{+} 1 \mathrm{H} \mathrm{NH}\right), 1.00(\mathrm{~d}, J=2.4 \mathrm{~Hz}, 3 \mathrm{H}), 0.97(\mathrm{~d}, J=2.4 \mathrm{~Hz}, 3 \mathrm{H}) .{ }^{13} \mathrm{C} \mathrm{NMR}(300 \mathrm{MHz}$, $\left.\mathrm{CDCl}_{3}\right) \delta: 175.22,154.41,136.51,136.00,128.65,128.04,126.83,115.11,108.23,91.90,73.15$, $66.42,51.60,45.45,31.72,25.88,19.18,18.56$

B-P10: $17 \%$. IR spectrum $\left(\mathrm{KBr}, \mathrm{cm}^{-1}\right) 698(\mathrm{C}-\mathrm{Cl}) .{ }^{1} \mathrm{H} \mathrm{RMN}\left(\mathrm{CDCl}_{3}, 300 \mathrm{MHz}\right), \delta$, ppm $(\mathrm{J}, \mathrm{Hz})$ : $\delta 7.42-7.30(\mathrm{~m}, 5 \mathrm{H}), 6.89(\mathrm{~d}, J=3.3 \mathrm{~Hz}, 1 \mathrm{H}), 6.50(\mathrm{~s}, 1 \mathrm{H}), 6.28(\mathrm{~d}, J=3.3 \mathrm{~Hz}, 1 \mathrm{H}), 3.86(\mathrm{~s}$, 2H), $3.73(\mathrm{~s}, 3 \mathrm{H}), 3.71(\mathrm{~s}, 2 \mathrm{H}), 3.11(\mathrm{~d}, J=5.7 \mathrm{~Hz}, 1 \mathrm{H}), 1.98\left(\mathrm{~h}, J=13.4,6.7 \mathrm{~Hz}, 1 \mathrm{H}^{+} 1 \mathrm{H} \mathrm{NH}\right)$, $0.99(\mathrm{~d}, J=2.4 \mathrm{~Hz}, 3 \mathrm{H}), 0.96(\mathrm{~d}, J=2.4 \mathrm{~Hz}, 3 \mathrm{H}) .{ }^{13} \mathrm{C} \mathrm{NMR}\left(300 \mathrm{MHz}, \mathrm{CDCl}_{3}\right) \delta: 175.22$, $152.57,149.70,137.10,131.51,129.16,128.65$, 127.08, 116.16,111.10, 109.37, 66.26, 51.59, $46.65,31.69,25.88,19.10,18.60$

\section{4-Methyl-2-\{[5-(3-phenyl-prop-1-ynyl)-furan-2-yl-methyl]-amino\}-pentanoic acid methyl ester (IM 11)}

Leucine methyl ester hydrochloride gave a mixture of IM11 and B-P11 as yellow oil form with a yield of $60 \%$

IM11: 73\%. IR spectrum $\left(\mathrm{KBr}, \mathrm{cm}^{-1}\right) 3336(\mathrm{~N}-\mathrm{H}), 2230(\mathrm{C} \equiv \mathrm{C}), 1733(\mathrm{C}=\mathrm{O}$ ester $) .{ }^{1} \mathrm{H}$ NMR $(300$ $\left.\mathrm{MHz} \mathrm{CDCl}_{3}\right) \delta 7.42-7.27(\mathrm{~m}, 5 \mathrm{H}), 6.49(\mathrm{~d}, J=3.3 \mathrm{~Hz}, 1 \mathrm{H}), 6.20(\mathrm{~d}, J=3.3 \mathrm{~Hz}, 1 \mathrm{H}), 3.90(\mathrm{~s}$, $2 \mathrm{H}), 3.81(\mathrm{~s}, 2 \mathrm{H}), 3.75(\mathrm{~s}, 3 \mathrm{H}), 3.37(\mathrm{t}, J=7.3 \mathrm{~Hz}, 1 \mathrm{H}), 1.93(\mathrm{~s}, 1 \mathrm{H}), 1.84-1.71(\mathrm{~m}, J=13.1,6.5$ $\mathrm{Hz}, 1 \mathrm{H}), 1.53(\mathrm{t}, J=7.1 \mathrm{~Hz}, 2 \mathrm{H}), 0.96(\mathrm{~d}, J=1.9 \mathrm{~Hz}, 3 \mathrm{H}), 0.91(\mathrm{~d}, J=4.1 \mathrm{~Hz}, 3 \mathrm{H}) .{ }^{13} \mathrm{C} \mathrm{NMR}$ $\left(300 \mathrm{MHz}, \mathrm{CDCl}_{3}\right) \delta: 176.02,154.13,136.60,135.98,128.64,128.03,126.83,115.08,108.34$, $91.92,73.12,59.06,51.80,44.92,42.75,25.87,24.86,22.75,21.20$

B-P11: 27\%. IR spectrum $\left(\mathrm{KBr}, \mathrm{cm}^{-1}\right) 698(\mathrm{C}-\mathrm{Cl}) .{ }^{1} \mathrm{H}$ NMR $\left(300 \mathrm{MHz}, \mathrm{CDCl}_{3}\right) \delta 7.42-7.27$ $(\mathrm{m}, 5 \mathrm{H}), 6.49(\mathrm{~d}, J=3.3 \mathrm{~Hz}, 1 \mathrm{H}), 6.48(\mathrm{~s}, 1 \mathrm{H}) 6.28(\mathrm{~d}, J=3.3 \mathrm{~Hz}, 1 \mathrm{H}), 3.86(\mathrm{~s}, 2 \mathrm{H}), 3.72(\mathrm{~s}, 2 \mathrm{H})$, $3.74(\mathrm{~s}, 3 \mathrm{H}), 3.37(\mathrm{t}, J=7.3 \mathrm{~Hz}, 1 \mathrm{H}), 1.93(\mathrm{~s}, 1 \mathrm{H}), 1.84-1.71(\mathrm{~m}, J=13.1,6.5 \mathrm{~Hz}, 1 \mathrm{H}), 1.50(\mathrm{t}$, $J=7.1 \mathrm{~Hz}, 2 \mathrm{H}), 0.96(\mathrm{~d}, J=1.9 \mathrm{~Hz}, 3 \mathrm{H}), 0.91(\mathrm{~d}, J=4.1 \mathrm{~Hz}, 3 \mathrm{H}) .{ }^{13} \mathrm{C} \mathrm{NMR}\left(300 \mathrm{MHz}, \mathrm{CDCl}_{3}\right)$ 
$\delta: 176.02,152.28,149.78,137.08,131.60,129.17,128.64,127.08,116.14,111.8,109.47,58.97$, $51.80,46.64,42.75,25.87,24.88,22.71,21.23$

\section{[5-(3-Phenyl-prop-1-ynyl)-furan-2-ylmethyl]-(3-tri-fluoroomethyl-phenyl)-amine (IM 12)}

Yellow oil mixture was obtained using 3-trifluoromethyl aniline as a starting product. Efficiency $85 \%$

IM12: 70\% .IR spectrum $\left(\mathrm{KBr}, \mathrm{cm}^{-1}\right) 3421(\mathrm{~N}-\mathrm{H}), 2230(\mathrm{C} \equiv \mathrm{C}), 1616(\mathrm{C}-\mathrm{F}) .{ }^{1} \mathrm{H}$ NMR $(300 \mathrm{MHz}$, $\left.\mathrm{CDCl}_{3}\right) \delta 7.49-7.31(\mathrm{~m}, 6 \mathrm{H}), 7.05(\mathrm{dd}, J=7.7,0.5 \mathrm{~Hz}, 1 \mathrm{H}), 6.96-6.92(\mathrm{~m}, 1 \mathrm{H}), 6.86(\mathrm{dd}, J=$ 7.2, $4.5 \mathrm{~Hz}, 1 \mathrm{H}), 6.56(\mathrm{~d}, J=3.3 \mathrm{~Hz}, 1 \mathrm{H}), 6.29$ (d, $J=3.3 \mathrm{~Hz}, 1 \mathrm{H}), 4.38$ (s, 2H), 3.93 (s, 2H),

${ }^{19} \mathrm{~F}$ NMR $\left(282 \mathrm{MHz}, \mathrm{CDCl}_{3}\right)-62.70$ (s). ${ }_{13} \mathrm{C}$ NMR $\left(300 \mathrm{MHz}, \mathrm{CDCl}_{3}\right) \delta: 150.02,147.53,136.93$, $135.90,131.57,129.74,128.73,128.06,126.94,124.37,116.09,115.26,114.53,111.30,108.54$, $92.47,72.91,41.24,25.88$.

B-P12: $30 \%$. IR spectrum $\left(\mathrm{KBr}, \mathrm{cm}^{-1}\right) 696(\mathrm{C}-\mathrm{Cl}) .{ }^{1} \mathrm{H}$ NMR $\left(300 \mathrm{MHz}, \mathrm{CDCl}_{3}\right) \delta 7.49-7.31(\mathrm{~m}$, $6 \mathrm{H}), 7.05(\mathrm{dd}, J=7.7,0.5 \mathrm{~Hz}, 1 \mathrm{H}), 6.96-6.94(\mathrm{dd}, J=7.2,4.5 \mathrm{~Hz}, 1 \mathrm{H}), 6.94\left(\mathrm{~m}, 1 \mathrm{H}^{+} 1 \mathrm{H}\right.$ furano), 6.51(s,1H), $6.38(\mathrm{~d}, J=3.3 \mathrm{~Hz}, 1 \mathrm{H}),, 4.38(\mathrm{~s}, 2 \mathrm{H}), 3.85(\mathrm{~s}, 2 \mathrm{H}) .{ }^{19} \mathrm{~F} \mathrm{NMR}(282 \mathrm{MHz}$, $\left.\mathrm{CDCl}_{3}\right)-62.70$ (s). ${ }^{13} \mathrm{C} \mathrm{NMR}\left(300 \mathrm{MHz}, \mathrm{CDCl}_{3}\right) \delta: 152.47,150.02,147.65,137.04,132.75$, 129.74, 129.20, 128.79, 127.17, 124.37, 116.09, 115.92, 114.53, 111.30, 109.4, 109.3, 41.20, 27.06 .

\subsection{Molecular docking}

Molecular docking is a very interesting means for predicting enzyme-ligand interactions and plays a very important role in the design of new molecules with therapeutic effect.

\subsubsection{Mature Streptococcalcysteine proteasem SpeB}

These results show that the complex formed between $\mathrm{mSpeB}$ and the IM9 analogue has the lowest energy $(-6.32288551 \mathrm{Kcal} / \mathrm{mol})$ and was more active than the complexes formed with the other analogues as well as with Carlina oxide (Table 2).

For complexes mSpeB-CarOx, mSpeB-IM10, mSpeB-IM12: except for the Van der Waals interactions (VDW) which are perceptible, the ligands do not show any other interaction. For the complex mSpeB- IM8, mSpeB- IM9 and mSpeB-IM11: the analogues interact with the amino acid HIS 195 pi-cation at a distance of 4.59, 4.65 and 4.81 A respectively (Fig 2, Table 2). 
Streptococcus pyogenesis one of the most common human pathogens. Less than $10 \%$ of normal individuals harbor the bacteria, usually in the respiratory tract and skin without any sign of disease [29]. In recent years, the variety, severity and sequelae of Streptococcus pyogenesis infections have increased; in addition, due to the occurrence of occasional cases of a rapidly progressive and incurably fatal disease, this pathogen is now a major preoccupation. Streptococcus pyogenesis escapes the human immune system and causes benign diseases such as pharyngitis and scarlet fever as well as septicaemia but also life-threatening. In addition, mild infections at the beginning can lead to serious complications such as rheumatic fever, to which serious heart infections can result if left untreated. It escapes the human immune system by cleavage of the antibody IgG from its surface, mediated by a cysteine protease [29,30]. A cysteine protease is an enzyme that catalyzes the hydrolysis of peptide bonds in other words, the peptide chains are cleaved, mediated by a nucleophilic thiol group present at the active site. However, cysteine protease can improve the ability of GAS to invade respiratory epithelial cells. To prevent such cleavage, there may be advantages to getting access to a protease inhibitor $[31,32]$.

\subsubsection{Dihydrofolate reductase DHFR from Enterococcus faecalis}

As a result, the complex formed between DHFR and the IM9 analogue has the lowest energy ($7.4878 \mathrm{Kcal} / \mathrm{mol}$ ) and was more active than the complexes formed with the other analogues as well as with Carlina oxide. For the complexes DHFR-CarOx, DHFR-IM9, DHFR- IM10 and DHFR- IM11: the ligands show no interaction, however Van der Waals (VDW) interactions are perceptible. For the DHFR- IM8 complex: the analogue interacts with the amino acid ARG 44 $\mathrm{H}$-acceptor, 2 different sites of SER $65 \mathrm{H}$-acceptor and THR $126 \mathrm{pi}-\mathrm{H}$ at a distance of 3.31, 3.13, 2.98 and $4.04 \AA$ respectively. For the DHFR- IM12 complex: the analogue interacts with the amino acid ARG $44 \mathrm{H}$-acceptor and THR 126 pi-H at a distance of 3.00 and $3.97 \AA$ respectively (Fig 3). The enzyme Dihydrofolate reductase (DHFR) was first discovered during the investigation on the mechanism of action of methotrexate for cancer treatment [32]. This enzyme is the only source of 5,6,7,8 tetrahydrofolate (THF); it promotes the NADPH-dependent reduction of 7,8-dihydrofolate giving 5,6,7,8 tetrahydrofolate, which is involved in the synthesis of nucleic acids and protein [33,34]. Inhibition of this enzyme blocks DNA synthesis and cell division, leading to cell death. Due to its importance, DHFR is the target of several important anti-cancer and antibacterial drugs [32-35]. 
Table 2: Energy balance of different complexes (enzyme-ligand) Kcal/mol

\begin{tabular}{ccccccc}
\hline & \multicolumn{2}{c}{ 2UZJ } & \multicolumn{2}{c}{ 4M7U } & \multicolumn{1}{c}{ 1KZN } \\
\hline & Score & Rmsd-refine & Score & Rmsd-refine & Score & Rmsd-refine \\
\hline Ligref & -6.3298 & 1.5951 & -7.9014 & 2.6476 & -8.4386 & 2.3946 \\
\hline CarOx & -4.8777 & 1.2085 & -4.6940 & 1.2917 & -5.4299 & 0.9631 \\
\hline Im8 & -5.9333 & 2.1819 & -5.8233 & 1.5904 & -6.1237 & 1.8091 \\
\hline Im9 & $\mathbf{- 6 . 3 2 2 9}$ & 1.6668 & $\mathbf{- 7 . 4 8 7 8}$ & 2.2864 & $\mathbf{- 6 . 6 7 4 9}$ & 1.6862 \\
\hline Im10 & -6.1176 & 2.5474 & -5.8177 & 2.4351 & -6.0193 & 1.7480 \\
\hline Im11 & -6.2669 & 1.5162 & -7.3378 & 1.2609 & -6.6611 & 1.6829 \\
\hline Im12 & -5.6369 & 2.2597 & -6.6581 & 2.2953 & -6.1715 & 1.5337 \\
\hline
\end{tabular}

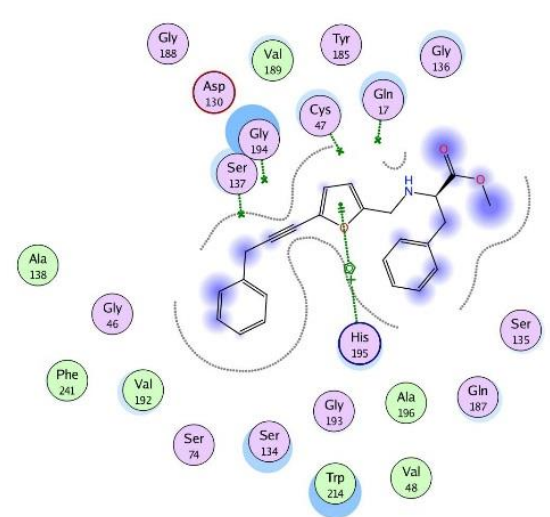

$\mathbf{a}$

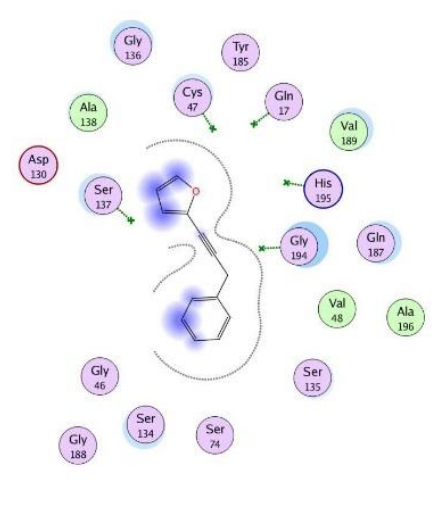

b

Fig 2: Diagrams of interactions enzyme-ligands: (a) diagram interaction of complexe mSpeB- IM9, (b) diagram interaction of $\mathrm{mSpeB}-\mathrm{CarOx}$

\subsubsection{DNA gyrase}

According table 2 the complex formed between DNA gyrase and the IM9 analogue has the lowest energy $\quad(-6.6749 \mathrm{Kcal} / \mathrm{mol})$ and was more active than the complexes formed with the other analogues as well as with Carlina oxide. For complexes formed with DNA gyraseCarOx, IM9 and IM10 with the only exception of Van der Waals interactions (VDW) which were noticeable, the ligands do not exhibit any other interaction. For the complex DNA gyrase-IM8: the analogue interacts with the amino acid ASN $46 \mathrm{H}$-donor at a distance of $3.28 \AA$. For the 
complex DNA gyrase-IM11: the analogue interacts with the amino acid PRO 79 pi-H at a distance of $4.05 \AA$.

For the complex DNA gyrase-IM12: the analogue interacts with 2 different sites of the amino acid ARG 76 pi-cation at a distance 10.97 and $12.93 \AA$ (Table 2, Fig 4). DNA gyrase is a main bacterial protein that is implicated in replication and transcription and catalyzes the negative supercoil of circular bacterial DNA. This topoisomerase type II is found in all bacteria. It has been isolated from many bacterial species, but the best characterized gyrase is Escherichia coli. DNA gyrase is a known as an effective target for antibacterial agents, since its blockingin duces bacterial death. Quinolones, coumarins, and cyclothialidins are known to have an inhibitory action on this enzyme. Mechanistic experiments and research on gyrase-specific drugs were conducted simultaneously and have proved to be of high interest [36,37].

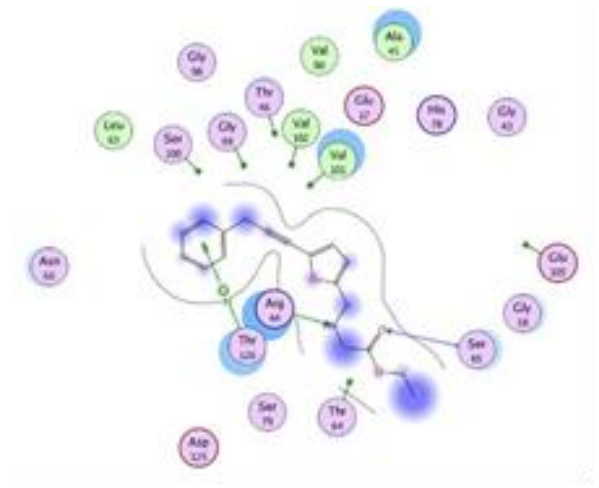

a

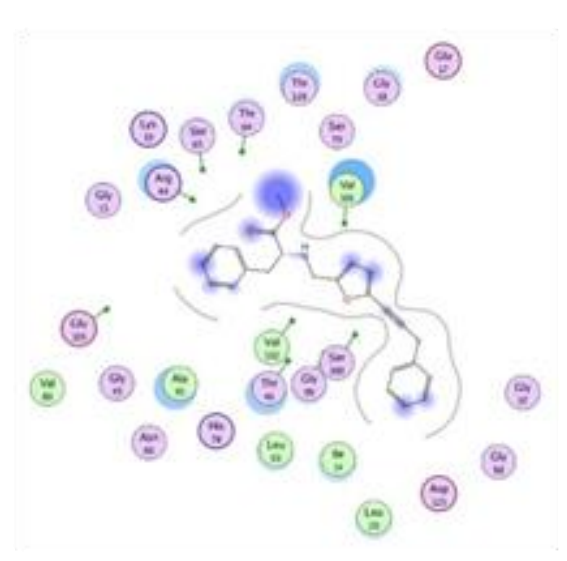

b

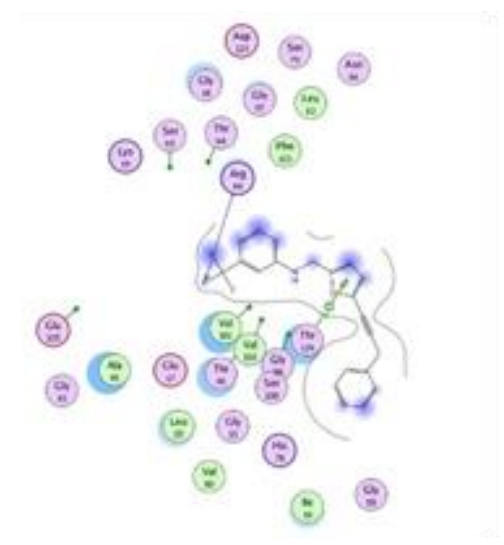

C

Figure 3: Diagrams of interactions (enzyme-ligands): (a): Diagram interaction of complexe DHFR- IM8; (b):

Diagram interaction of complexe DHFR- IM9; (C): Diagram interaction of complexe DHFR- IM12 


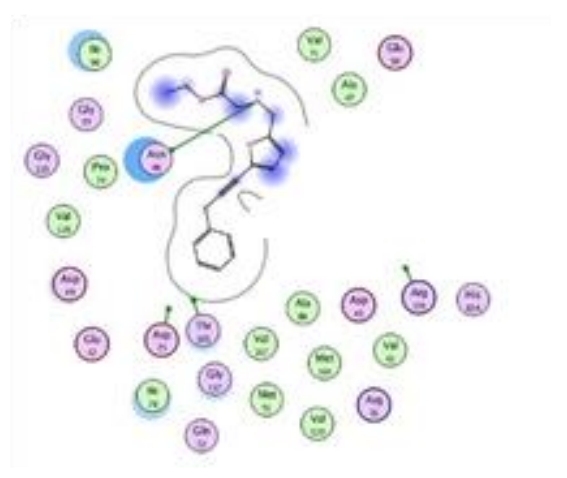

(a)

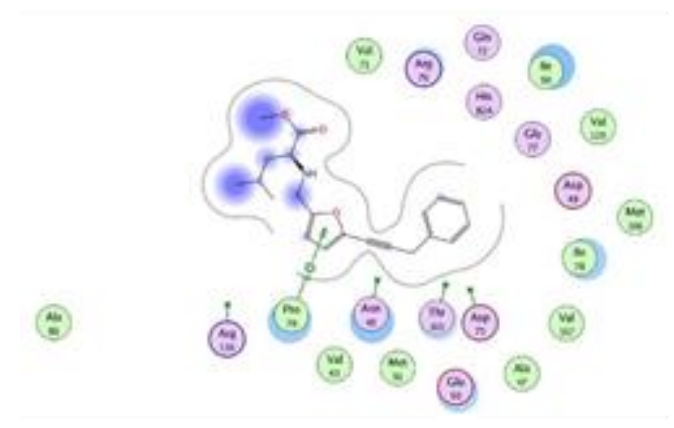

(c)

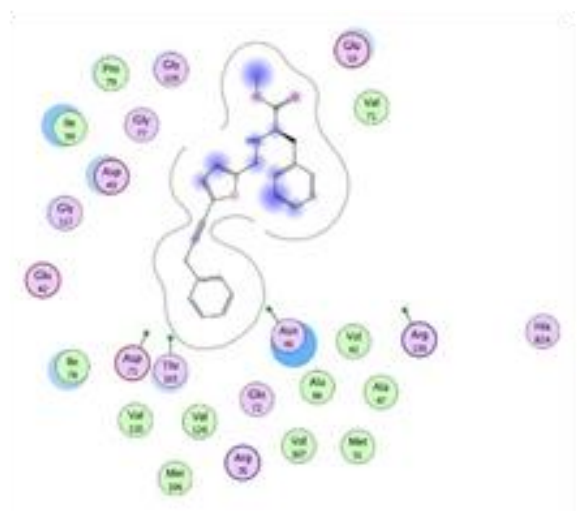

(b)

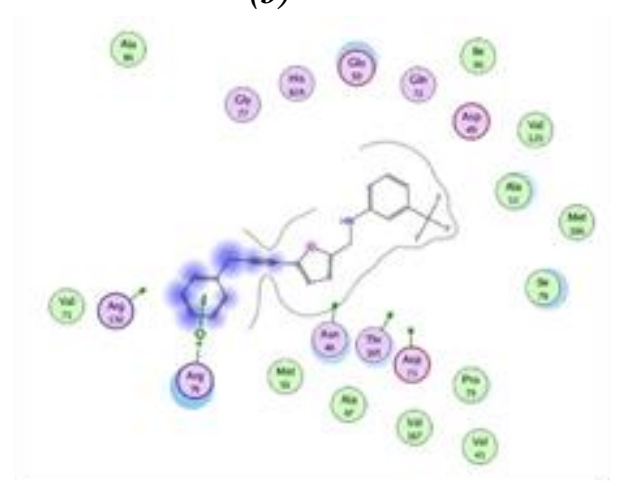

(d)

Fig 4: Diagrams of interactions (enzyme-ligands): (a): diagram interaction of complexe DNAgyrase- IM8; $b$ : diagram interaction of complexe DNAgyrase- IM9; (c): diagram interaction of complexe DNAgyrase- IM11; (d) : diagram interaction of complexe DNAgyrase- IM12

\section{Conclusion}

In conclusion, we have been able to introduce some modifications to the structure of Carlina oxide while keeping the triple bond, thus, creating new analogues through known chemical pathways. The structures of the new compounds have been confirmed by different spectroscopic methods. Molecular docking have also predicted an interesting inhibitory activity of three enzymes of bacterial origin of almost all analogues, mainly IM9 compared to Carlina oxide which is known for its antibacterial properties, and which also helps to understand in detail the various interactions between ligands and enzyme active sites in order to help design new potent inhibitors.

\section{ETHICS APPROVAL AND CONSENT TO PARTICIPATE}

Not applicable.

\section{HUMAN AND ANIMAL RIGHTS}


No Animals/Humans were used for studies that are the basis of this research.

\section{CONSENT FOR PUBLICATION}

Not applicable.

\section{RESEARCH INVOLVING PLANTS}

The authors declare that species studied is not endangered.

\section{AVAILABILITY OF DATA AND MATERIALS}

Not applicable.

\section{FUNDING}

None.

\section{CONFLICT OF INTEREST}

The authors declare no conflict of interest, financial or otherwise.

\section{ACKNOWLEDGEMENTS}

The authors are indebted to Benabadji $\mathrm{N}$ from University of Tlemcen, Laboratory of Ecology and Management of Natural Ecosystems, to botanical identification of plants.

\section{References}

(1) Oliveira, C. S. de; Lira, B. F.; Barbosa-Filho, J. M.; Lorenzo, J. G. F.; Menezes, C. P. de; Santos, J. M. C. G. dos; Lima, E. de O.; Athayde-Filho, P. F. de. Synthesis and Testing of 3Acetyl-2,5-Disubstituted-2,3-Dihydro-1,3,4-Oxadiazole Derivatives for Antifungal Activity against Selected Candida Species. J. Braz. Chem. Soc. 2013, 24 (1), 115-120. https://doi.org/10.1590/S0103-50532013000100016.

(2) Arnold L. Demain; Preeti Vaishnav. Natural Products for Cancer Chemotherapy. Microb Biotechnol 2011, 4 (6), 687-699. https://doi.org/10.1111/j.1751-7915.2010.00221.x.

(3) Li J; Zhang C; Gong M; Wang M. Combination of Artemisinin-Based Natural Compounds from Artemisia Annua L. for the Treatment of Malaria: Pharmacodynamic and Pharmacokinetic Studies. Phytother Res 2018, 32 (7), 1415-1420. https://doi.org/10.1002/ptr.6077. 
(4) Yue-Zhong Shu. Recent Natural Products Based Drug Development: A Pharmaceutical Industry Perspective. J. Nat. Prod. 1998, 1053-1071.

(5) Gupta, V. K. Comprehensive Bioactive Natural Products; Stadium Press: Texas, 2010.

(6) Gottlieb, D.; Shaw, P. D. Biosynthesis; 1967.

(7) Ashworth, P. J..; Jones, E. R. H.; Mansfield, G. H.; Schlögl, K.; Thompson, J. M.; Whiting, M. C. Researches on Acetylenic Compounds. Part LIX. The Synthesis of Three Polyacetylenic Antibiotics. J. Chem. Soc 1958, 950-954. https://doi.org/10.1039/jr9580000950.

(8) Konovalov, D. Polyacetylene Compounds of Plants of the Asteraceae Family. Pharm. Chem. J. 2014, Vol. 48 (9), 615. https://doi.org/10.1007/s11094-014-1159-7.

(9) Minto, R. E; Blacklock, B. J. Biosynthesis and Function of Polyacetylenes and Allied Natural Products. Prog. Lipid Res. 2008, 47 (4), 233-306. https://doi.org/10.1016/j.plipres.2008.02.002.

(10) Chang Zeng Wang; De Quan Yu. LIGNAN AND ACETYLENIC GLYCOSIDES FROM ASTER AURICULATUS. Phytochemistry 1998, 48 (4), 711-717. https://doi.org/10.1016/s0031-9422(98)00019-3.

(11) Christensen, L. P., \& Lam, J. Acetylenes and Related Compounds in Cynareae. Phytochemistry 29 (9), 2753-2785. https://doi.org/10.1016/0031-9422(90)87075-6.

(12) Shi Shun, A. L. K.; Tykwinski, R. R. Synthesis of Naturally Occurring Polyynes. Angew. Chem. Int. Ed. 2006, 45 (7), 1034-1057. https://doi.org/10.1002/anie.200502071.

(13) Shi Shun, A. L. K.; Tykwinski, R. R. Synthesis of Naturally Occurring Acetylenes via an Alkylidene Carbenoid Rearrangement. J. Org. Chem. 2003, 68 (17), 6810-6813. https://doi.org/10.1021/jo034734g.

(14) Semmler, F. W. Zusammenseteung Des Atherisohen Oelg Der Eberwurzel (Carlina Acaulis L.). 1906.

(15) GILMAN, H.; VAN Ess, P. R.; BURTNER, R. R. The Constitution of Carlina-Oxide. $J$. Am. Chem. Soc 1933, 55 (8), 3461-3463. https://doi.org/10.1021/ja01335a075.

(16) Eicher, T.; Hauptmann, S.; Speicher, A. The Chemistry of Heterocycles: Structure, Reactions, Syntheses, and Applications, 2nd, completely rev., and enl. ed ed.; Wiley-VCH: Weinheim, 2003. 
(17) Maciej Strzemski; Magdalena Wójciak-Kosior; Ireneusz Sowa; Monika Agacka-Mołdoch; Piotr Drączkowski; Dariusz Matosiuk; Łukasz Kurach Ryszard Kocjan; Sławomir Dresler. Application of Raman Spectroscopy for Direct Analysis of Carlina acanthifolia Subsp. Utzka Root Essential Oil. Elsevier BV 2017, 1 (174), 633-637. https://doi.org/10.1016/j.talanta.2017.06.070.

(18) Bohlmann, F.; Schuster, A.; Meusel, H. A Carlina Oxide Derivative from Carlina Diae. Phytochemistry 1981, 20 (4), 823-824. https://doi.org/10.1016/0031-9422(81)85186-2.

(19) Belabbes, R.; Mami, I. R.; Dib, M. E. A.; Mejdoub, K.; Tabti, B.; Costa, J. Chemical Composition and Biological Activities of Essential Oils of Echinops Spinosus and Carlina vulgaris Rich in Polyacetylene Compounds. Curr. Nutr. Food Sci. 2019, 15, 1-8.

(20) Mejdoub, K.; Mami, I. R.; Belabbes, R.; Dib, M. E. A.; DJabou, N.; Tabti, B.; Benyelles, N. G.; Costa, J.; Muselli, A. Chemical Variability of Atractylis gummifera Essential Oils at Three Developmental Stages and Investigation of Their Antioxidant, Antifungal and Insecticidal Activities. Curr. Bioact. Compd. 2019, 15.

(21) Mami, I. R.; Belabbes, R.; Dib, M. E. A.; Tabti, B.; Costa, J.; Muselli, A. Biological Activities of Carlina Oxide Isolated from the Roots of Carthamus caeruleus. Nat. Prod. J. 2019, 9, 1-8.

(22) Herrmann, F.; Hamoud, . Razan; Sporer, F.; Tahrani, A.; Wink, M. Carlina Oxide - A Natural Polyacetylene from Carlina acaulis (Asteraceae) with Potent Antitrypanosomal and Antimicrobial Properties. Planta Med 2011, 77, 1905 - 1911.

(23) Đordevic, S.; Petrovi'c, S.; Dobri'c, S.; Milenkovi'c, M.; Vu 'ci'cevi'c, D.; Zizi'c, S.; Kuki'c, J. Antimicrobial, Anti-Inflammatory, Anti-Ulcer and Antioxidant Activities of Carlina acanthifolia Root Essential Oil. J. Ethnopharmacol. 2007, 109, 458-463.

(24) Link, P.; Roth, K.; Sporer, F.; Wink, M. Carlina Acaulis Exhibits Antioxidant Activity and Counteracts A $\beta$ Toxicity in Caenorhabditis elegans. Molecules 2016, 21 (7).

(25) Benelli, G.; Pavela, R.; Petrelli, R.; Nzekoue, F. K.; Cappellacci, L.; Lupidi, G.; Quassinti, L.; Bramucci, M.; Sut, S.; Dall'Acqua, S.; et al. Carlina Oxide from Carlina acaulis Root Essential Oil Acts as a Potent Mosquito Larvicide. Ind. Crops Prod. 2019, 137, 356-366. https://doi.org/10.1016/j.indcrop.2019.05.037.

(26) Pavela, R.; Maggi, F.; Petrelli, R.; Cappellacci, L.; Buccioni, M.; Palmieri, A.; Canale, A.; Benelli, G. Outstanding Insecticidal Activity and Sublethal Effects of Carlina acaulis Root 
Essential Oil on the Housefly, Musca domestica, with Insights on Its Toxicity on Human Cells. Food Chem. Toxicol. 2020, 136, 111037. https://doi.org/10.1016/j.fct.2019.111037.

(27) Saffidine, K.; Sahli, F.; Zerroug. Antimicrobial Activity of an Algerian Medicinal Plant: Carthamus caeruleus L. 6.

(28) Molecular Operating Environment (MOE); Chemical Computing Grouplnc: Canada, 2013.

(29) Olsen, J. G.; Dagil, R.; Niclasen, L. M.; Sørensen, O. E.; Kragelund, B. B. Structure of the Mature Streptococcal Cysteine Protease Exotoxin MSpeB in Its Active Dimeric Form. $J$. Mol. Biol 2009, 393, 693 - 703.

(30) BERGGREN, K. Synthesis of Novel Inhibitors of IdeS, a Bacterial Cysteine Protease Including Studies of Stereoselective Reductive Aminations. 2011.

(31) TSAI, P.-J.; KUO, C.-F.; LIN, K.-Y.; LIN, Y.-S.; LEI, H.-Y.; CHEN, F.-F.; WANG, J.-R.; WU, J.-J. Effect of Group A Streptococcal Cysteine Protease on Invasion of Epithelial Cells. Am. Soc. Microbiol. 1998, 66 (4), 1460-1466.

(32) Hawser, S.; Lociuro, S.; Islam, K. Dihydrofolate Reductase Inhibitors as Antibacterial Agents. Biochem. Pharmacol. 2006, 71, 941-948.

(33) Antypenko, L. M.; Kovalenko, S. I.; Los, T. S.; Rebec, O. L. Synthesis and Characterization of Novel N-(Phenyl, Benzyl, Hetaryl)-2-([1,2,4]Triazolo[1,5-c]Quinazolin-2-ylthio) Acetamides by Spectral Data, Antimicrobial Activity, Molecular Docking and QSAR Studies. J. Heterocycl. Chem. 2017, 54 (2), 1267-1278.

(34) Bourne, C. R.; Wakeham, N.; Webb, N.; Nammalwar, B.; Bunce, R. A.; Berlin, K. D. The Structure and Competitive Substrate Inhibition of Dihydrofolate Reductase from Enterococcus Faecalis Reveal Restrictions to Cofactor Docking. Biochemistry 2014, 53, $1228-1238$.

(35) Oefner, C.; Bandera, M.; Haldimann, A.; Laue, H.; Schulz, H.; Mukhija, S.; Parisi, S.; Weiss, L.; Lociuro, S. Increased Hydrophobic Interactions of Iclaprim with Staphylococcus aureus Dihydrofolate Reductase Are Responsible for the Increase in Affinity and Antibacterial Activity. 2009, 63, 687-698.

(36) Maxwell, A. DNA Gyrase as a Drug Target. Trends Microbiol 1997, 5 (3), 102-109.

(37) Lafitte, D.; Lamour, V.; Tsvetkov, P. O.; Makarov, A. A.; Klich, M.; Deprez, P.; Moras, D.; Briand, C.; Gilli, R. DNA Gyrase Interaction with Coumarin-Based Inhibitors: The Role of 
the Hydroxybenzoate Isopentenyl Moiety and the 5'-Methyl Group of the Noviose. Biochemistry 2002, 41, 7217-7223. 